\title{
Review of Energy Harvesting Techniques for Wireless Sensor Nodes
}

\author{
Anuj Garg \\ Dept. of Elec. \& Comm. LNCT, \\ Bhopal, India.
}

\author{
R. K. Baghel \\ Dept. of Elec. \& Comm. MANIT, \\ Bhopal, India.
}

\author{
Mukesh Pandey \\ School of Energy \& Env.Mgt \\ UTD,RGPV, Bhopal,India.
}

\begin{abstract}
As demand of energy, power costs, and emission of carbon continue to increase, there is a need to control energy use to save environment and resources. To manage these issues, a novel, plug-through power directing framework for business and homes is required. With usage of wireless sensors, the management of their energy resources has turned into a subject of research.Wireless sensors expectedly utilize batteries as their power supply however in a few applications battery replacement can be inelegant and require more time which can influence the procedure being outwardly regulated. It is conceivable to collect energy from the sources in nature for wireless sensors. Here energy sources has been demonstrated keeping in mind the end goal to manage their cumulation of various things together that work as one unit with wireless sensor systems.
\end{abstract}

\section{Keywords}

Wireless sensor, energy harvesting, nodes, network, system.

\section{INTRODUCTION}

Wireless Sensor Networks (WSNs) have had cosmically huge influence in the examination field of multi-hopwireless systems as empowering agents of Personal Computer projects going from related to evading conditions and makes it overwhelming outwardly administering to fringe security and human wellbeing control. Research inside this field has secured a wide range of subjects, prompting progresses in node equipment, standards of benergy harvesting avior stack configuration, limitation and optically soliciting and taking after methods for doing things and energy management [1]. Research on WSNs has been driven and possibly repressed by a common focus: Energy eficiency. Nodes of a WSN are expectedly controlled by electrical storage contraptions. Once their energy is used up, the node is dead. Only in extremely specific applications electrical storage contraptions can be superseded or revived. Be that as it may, not with standing when this is conceivable, the replacement operation is moderate and lavish, and diminishes system performance. Different methods for doing things have consequently been proposed to decelerate the using everything up completely of electrical storage contraption energy, which incorporate power control and the usage of duty cycle-predicated operation. The exact opposite thing just specified method for doing things utilizes low power methods of wireless handsets, whose segments can be exchanged off for energy protecting. At the point when the node is in a low power (or sleep) mode its utilization is much lower than when the handset is ON [2, $3]$. In any case, when sleeping the node can't transmit or receive bundles. The commitment cycle communicates the proportion between the time when the node is on and the entirety of the times when the node is $\mathrm{ON}$ and sleeping. Embracing principles of benergy harvesting avior that work at very low commitment cycles is the main kind of answer for empowering lasting WSNs [4]. However, this approach experiences two primary impacts. 1) There is an implicit exchange off between energy (i.e., low commitment cycling) and information deferral, and 2) battery worked WSNs don't give the required response to the required things of some from early on noticeable applications that interest system lifetimes of no less than 20 years or more. Electrical storage creation spillage goes through electrical storage inventions inside a few years regardless of the possibility that they are for all intents and purposes never utilized $[5,6]$. Therefore late research on enduring WSNs is taking an alternate methodology, proposing energy gatherers cumulated with the use of rechargeable electrical storage contraptions and super capacitors as the key enabler to steady WSN operations. Energy storing up predicated WSN are the consequence of giving WSN nodes with the office of taking from something else energy from the evading. Energy accumulating can plenarily use diffeerent wellsprings of energy, for example, solar powered power, wind, mechanical vibrations, temperature variations, attractive fields, etc. Continuously giving energy, and putting away it for future use, energy storing subsystems empower WSN nodes to last potentially for ever. This part investigates the opportunities and difficulties of energy harvesting WSNs, clarifying why the outline of tenets of benergy harvesting avior stacks for traditional WSNs must be plenarily returned to.

\section{RELATED WORK}

Wireless sensor systems (WSNs) lies on the top when we consider its nature of being savored a ton, it is conceivable because of its beginning and interesting and intriguing PC programs in for all intents and purposes all fields going from environment managing to battlefield circumstance. In key advances that run a PC, it sets a a new level for monitoring different environments. It worried to geographical areas related to where mountains, streams, urban areas, and so on., are located and where human activity that profits a wretched circumstance is impractical [7, 8, 9]. Every sensor node in WSN comprises of four simple units in particular sensor unit, handset unit, processorunit, power unit. The work of the sensor unit is to sense the specific conditions, for example, temperature, weight level, humiditylevel and so forth., again this detected auxiliary data changed over to advanced sign for communication purpose through an A-D converter. The processor unit incorporates a microcontroller and memory unit. It safeguards the detected information. The handset unit makes up of wireless radio transmitters and collector stations. The power unit comprises of electrical storage contraptions that provide mandatory energy to different units $[10,11]$. Numerous standards of benergy harvesting avior proposed to decrease the energy use and in that way build the lifetime of the sensor system. These guidelines of conduct separated and 
named into three sorts. The tenets of benergy harvesting avior going under top of the line category focuses on controlling of transmission power at node level and in the meantime keep up the connectivity of the system; it is conceivable by augmenting the system office to accomplish something. Be that as it may, here we need to agree with the expense of the system. Principles of benergy harvesting avior alluded to next class settle on directing choices predicated upon power advancement objectives. At that point guidelines of benergy harvesting avior incorporated into the last class settle on very important and auxiliary choices about topology control, that connotes which sensor nodes take partin the system action and which are not sleep mode participating in the network operations simultaneously $[12,13,14,15]$. Here the nodes have the great intellect about their (relating to every individual or thing) positions through GPS or message passing.

Bunching methods for doing things connected to lessen the energy use. These methods for improving burden adjusting, utilizable important supply use and information harvesting. As from examination of various papers, numerous guidelines of conduct propose toward energy use on transfer ways to addition energy. Some proposes transmuting directing principles of benergy harvesting avior in this sizably voluminous picture. Anyway, these methodologies are not giving great results when a few nodes are present in the most handing-off way, it alludes to well known way. As a consequence of this directing circle and unlimited delay are comes into picture. This grouping approach does not give any originations about node offices and node conveyance in the system. In the exemplary distributed comparison, a node can either cut or source, however both can't be conceivable. At the point when energy backup profitable supply of a node spent, that node is, a dead node or it does not communicate. This is the explanation benergy harvesting ind turning the server part among every one of the nodes present in the network. The exploration works with respect to WSN in the monstrously enormous picture of correspondence give a new direction to directing guidelines of benergy harvesting avior. Which has given focus on to separated the courses for data transmission from the sensor node to base station in a manner that, the system lifetime. It is not an everlasting answer for the lifetime of the network, it is auxiliary imperceptibly. As we ken sensor nodes are electrical storage contraption powered, so outlining of best directing tenets of benergy harvesting avior plays a cosmically principal part in WSN. Since electrical storage contraption, supersession is a colossally significant issue in the vast majority of the applications where sensor nodes conveyed and used in more numbers. So the exertion needs to make the sensor network in way, the nodes get their energy from it self where they conveyed and used. Thusly, it overcomes the limits. It spoke to in another way we may call it as energy. Energy storing up can be possible through power sources, for example, solar, thermal, mechanical radio recurrence and so forth. Any of these sources indicates time form properties like pulse based deportment and recurrence part variations. An unreasonably intriguing truth of harvesting energy is that, the harvesting exertion of harvesting source's random nature and correspondence process comes about the main going on for a brief timeframe using everything up of energy storage unit of sensor nodes. Some examination works discover great arrangement, by using piezoelectric materials for transmuting from one shape, state, or perspective to another of strain energy from a structure into electrical energy to gather energy to the sensor nodes in the network. This is a dumb founding and entrancing methodology $[16,17,18]$. It gives motivation to accomplish a considerable measure to work in this field. Numerous other individuals who work to discover data are also active around there. There are distinctive methods for achieving objectives to pick up power from the environment. At the point when rate of release is greater than the rate of charge then the specific framework will come up short on power. One interesting idea is that if the strength utilizes or control drawn by the sensing system then the injuctive approval of power output from the reaper diminished to more prevalent degree. To elucidate the perspective of energy utilize, the following energy use in WSN chart introduced here. The energy supplying arrangement of WSN incorporates three parts, for example, energy harvesting device, energy consuming device and energy storage device

The storage of the energy harvesting creation is to collect energy to control the sensor nodes in the system; it acts as producer. The part of the energy consuming device is to use the harvested energy for supporting diverse workloads like testing, correspondence and calculation $[19,20]$.The energy storage device, the part of storing aggregated energy from environment it gives energy to outer sensor nodes. It has double part; it can be either engenderer or individual according to the present circumstance.

\section{ENERGY HARVESTING METHODS IN WIRELESS SENSOR NODES}

Energy harvesting can be described as the procedure of record by a camera, accumulation and storage of energy from circumambient sources [21].The energy can be exuding from solar, temperature, motion or electromagnetic. RF wireless transmission of information is broadly settled in modern days, eg. cell systems, radio and telecom companies. Example: Even at a kilometer away from a FM radio tower, indoor power densities superior to anything $0.5 \mathrm{uW} / \mathrm{cm} 2$ can be distinguished. In light of this wide availability of wireless system measure of RF energy can be identified in the environment. With ideal hardware this energy can be adjusted to power self-decision sensor nodes. RF energy harvesting uses the power that would have generally been wasted and drowned up in the environment. Short range $(<2 \mathrm{~m})$ RFID PC programs focus on the mechanical science-medical(ISM) frequency bands around $0.9,2.4,5.8 \mathrm{Ghz}$ and higher. With the incrementation of recurrence, the wavelength decreases, making it useful for wireless self-decision transducer frameworks (WATS) [21]. The energy harvesting circuit ought to remain constantly dynamic to catch this particle of energy.The power usage of the reaper circuit should be minutely minor when contrasted with the energy given by the sources. Furthermore the energy harvesting circuit ought to be capable to store the aggregated energy with the base leakage [22]. Energy harvesting or power testing is most appropriate for applications that need continuous supply of low power, or the uses that need high power for a moment time span of time. Couple of conceivable energy hotspots for energy harvesting are as per the following:

\subsection{Solar Based}

A standout amongst the most prominent energy harvesting method for doing things is the usage of solar based cell. In this kind of energy collectors optical energy for the most part from daylight is changed over into electrical energy. Due to 
this it is most appropriate for uses that have sufficiently epic presentation to solar. The output of solar powered cell relies on upon the daylight and also on the heap added to it. Solar based cells have conversion efficiency upto $30 \%$. Solar based radiation causes around $15 \mathrm{~mW} / \mathrm{cm} 2$ in full solar light or $0.006 \mathrm{~mW} / \mathrm{cm} 2$ under blazing indoor illumination [22].

\subsection{Thermoelectric}

The era of power using a temperature is alluded to as thermoelectricity. A temperature difference between two junctions of a conducting material creates a conceivable distinction. This conceivable contrast is used by thermoelectric engenderers.

\subsection{Mechanical Vibration}

If an inertial mass is subjected to some movement electrical energy can be generated using three mechanisms: piezoelectric, electrostatic and electromagnetic [22].

\subsubsection{Piezoelectric}

Converts mechanical (weight, vibration) energy into electricity.

\subsubsection{Electrostatic}

The planes of at initially charged varactors are dissevered by vibration and the similar mechanical energy is used to incite electrical energy. One devoted voltage source is needed approved for this kind of energy era to charge the capacitors at first.

\subsubsection{Electromagnetic}

This is a backup method of energy harvesting which is free of the impacts of mechanical. Permanent magnets, coils and cantilever shaft are used for this sort of energy harvesting.But on account of its monstrously titanic size these are difficult to blend distinctive things together so they fill in as one unit with wireless sensor nodes.

\subsection{Far Field RF Energy Harvesting}

Far field RF energy harvesting should be possible in two ways: Active energy harvesting by using a devoted energy transmitter or energy harvesting using the wellsprings of energy present in evading conditions, for example, radio waves or solar light. One conceivable strategy for RF energy harvesting is by using television broadcast energy. Energy from RF business TV stations like TV or radio are used to supply energy to WSN. Energy is harvested using rectenna (rectifier +antenna) principle with a tuner stage. The tuner winnows one out of the possible commercial TV channels. The selected channel is the more puissant one and to this the sensor node is connected.

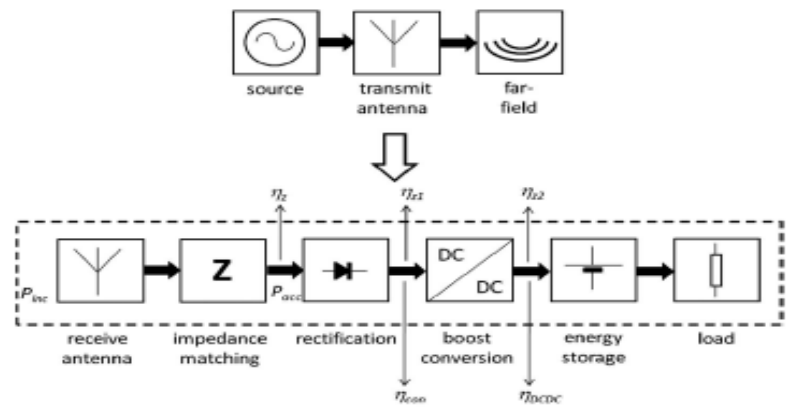

Fig. 1 Wireless RF power system, with the rectenna in the dashed box

\section{CONCLUSION}

An outline of the energy from wind, solar based, biomass, and so forth sources was displayed in this review article and the investigation using wind energy demonstrated the probability of this source to power low electronic devices. It is basic to note that frameworks for harvesting and putting away energies will be outlined predicated on the cumulation of two o rmore wellsprings of energy from wind, solar based, biomass, and so on. Case in point, for those electronics systems in the open air, solar based and wind energies can be accepted as sources for fueling the devices or on account of low power electronic contraptions found indoor, radio waves and Thermal energy can be separated. The mixture of two energy sources expand the shot of having uninterruptible power supply because every source can pay some shakiness of energy of its homogeneous individual or thing transmitted to the controlled invention. In additament to the unremarkable PC projects of energy harvesting, this innovation can be used in the origination of "Smart Homes" and additionally the management and driving of true protests in the Internet of Things [23]. With a specific end goal to revise the harvesting process using this sort of energy, the accompanying points can be considered in the future research-

1. Put into use a miniaturized scale wind turbine model. This characterizes a framework where one can enter an input parameter, for example, wind rate and cutting edge sweep and predicated on that the output power can be gotten.

2. Create cumulation of two things models with a specific end goal to victual the sensor from more than one source. Subsequently, more energy can be put away for the node and this prompts putting away more energy and in addition giving a more credible battery less operation of the general framework.

3. So as to secure of a never ending operation of the sensor, a cumulation of an energy storage arrangement and the harvesting framework may output as teady operation of the sensor node.

4. As far as applications, a framework made out of solarlight based cell and small scale wind turbine can be selected for pipeline regulating following superseding electrical storage inventions in these zones can take quite a while and stop the procedure. In a few ranges which have enough solarlight and wind, this model can be connected to give a never ending operation of sensors.

5. Thermal energy harvesting has moreover increased for fueling microelctronic devices. This innovation can be moreover used in pipeline monitoring as a source to power sensors in which case it is ready to use the temperature difference between the pipes and surrounding environment. The possibility of harvesting energy from nature is captivating yet the cost issues of conveying and using such an innovation will be meticulously thought accepted withal. A scientific approach to put into useing this element for low electronic applications needs to conclude the value, sum, or nature of it and choose if energy testing demonstrates better results in both specialized and economical aspects in correlation with different advancements. The exploration which has been doing apathetic microelectronics fields, for example, transmitter, 
microcontroller, storage schemes alongside the energy harvesting will exhibit all the more strong results in the future.

\section{REFERENCES}

[1] I. F. Akyildiz and M. C. Vuran. Wireless Sensor Networks. Advanced Texts in Communications and Networking. John Wiley \& Sons, Hoboken, NJ, August 2010.

[2] Moteiv Corporation. Datasheet Telos Rev B (Low Power Wireless Sensor Module),vMay 2004.

[3] Crossbow Technology, Inc. Datasheet MICAz, 2004.

[4] U. M. Colesanti, S. Santini, and A. Vitaletti. DISSsense: An adaptive ultralowpower communication protocol for wireless sensor networks. In Proceedings of IEEE DCOSS 2011.

[5] I. Buchmann. Batteries in a portable world: A handbook on rechargeable batter- ies for non-engineers. Cadex Electronics, Inc., second edition, May 2001.

[6] T. B. Reddy and D. Linden, editors. Linden's Handbook of Batteries. McGraw Hill, New York, NY, fourth edition, October 2010.

[7] Lee Myeong-Hyeon, Choi Yoon-Hwa. Fault detection of wireless sensor networks. Computer Communications 2008;31:3469-75

[8] Bari Ataul, et al. Design of fault tolerant wireless sensor networks satisfying survivability and lifetime requirements. Computer Communications 2012;35: 32033.

[9] Challal Y, et al. Secure and efficient disjoint multipath construction for fault tolerant routing in wireless sensor networks. Journal of Network and Compu- ter Applications 2011;34:1380-97.

[10] D.D. Geeta , N.Nalini , RajashekharC.Biradar. Fault tolerance in wireless sensor network using hand-off and dynamic power adjustment approach, Journal of Network and Computer Applications 36 (2013) 1174-1185

[11] B. Shirazi, A.R. Hurson, and K.M. Kavi, Scheduling and Load Balancing in Parallel and Distributed Systems. IEEE CS Press, 1995

[12] C. Intanagonwiwat, R. Govindan, and D. Estrin, "Directed Diffusion: A Scalable and Robust Communication Paradigm for Sensor Networks," Proc. ACM/IEEE Int'l Conf. Mobile Computing and Networking (MOBICOM), 2000.

[13] J. Kulik, W. Heinzelman, and H. Balakrishnan, "Negotiation- Based Protocols for Disseminating Information in Wireless Sensor Networks\}," ACM Wireless Networks, vol. 8, nos. 2-3, pp. 169-185, 2002. citeseer.nj.nec.com/kulik99negotiationbased. Html.
[14] J.-H. Chang and L. Tassiulas,"Energy Conserving Routing in Wireless Ad-Hoc Networks," Proc. IEEE INFOCOM, Mar. 2000.

[15] W. Heinzelman, A. Chandrakasan, and H. Balakrishnan, "An Application-Specific Protocol Architecture for Wireless Microsensor Networks," IEEE Trans. Wireless Comm., vol. 1, no. 4, pp. 660- 670, Oct. 2002.

[16] K. Akkaya, M. Younis, A survey of routing protocols in wireless sensor networks, Elsevier Ad Hoc Network Journal 33 (2005) 325-349.

[17] V. Mhatre, C. Rosenberg, Energy and cost optimizations in wireless sensor networks: a survey, in: A. Girard, B. Sanso, F. Vazquez-Abad (Eds.), Performance Evaluation and Planning Methods for the Next Generation Internet, Kluwer Academic Publishers, 2005, pp. 1-23, chap. 1

[18] C.P. Townsend, S.W. Arms, Wireless Sensor Networks: Principles and Applications, Sensor Technology Handbook, Editor:Jon S. Wilson, publisher: Elsevier Newnes, ISBN: 0-7506-7729-5, Chapter 22, pp. 575-589, 2005

[19] Ossama Younis, Student Member, IEEE, and Sonia Fahmy, Member, IEEE HEED: A Hybrid, EnergyEfficient,Distributed Clustering Approach for Ad Hoc Sensor Networks.IEEE transactions on mobile computing, vol. 3, no. 4, october-december 2004.

[20] Z.J. Haas, M.R. Pearlman, and P. Samar, "The Zone Routing Protocol (ZRP) for Ad-Hoc Networks," Internet Draft, draft-ietfmanet-zone-zrp-04.txt, July 2002.

[21] Mai Ali, Lutfi Albasha, Nasser Qaddoumi Department Of Electrical Engineering ,American University Of Sharjah ,Sharjah, United Arab Emirates, "Rf Energy Harvesting For Autonomous Wireless Sensor Networks", Design And Technology Of Integrated Systems In Nanoscale Era (Dtis), 2013 8th International Conference, Digital Object Identifier 10.1109/Dtis.2013.6527782.

[22] Mile K. Stojčev1, Mirko R. Kosanović2, Ljubiša R. Golubović3, "Power Management And Energy Harvesting Techniques For Wireless Sensor Nodes", Telecommunication In Modern Satellite,Cable And Broadcasting Services,2009.Telsiks'09 9th International Conference: Digital Object Identifier:10.1109/Telsks.2009.5339410, Publication Year 2009,Page(S):65-72.

[23] D. Kelaidonis, A. Somov, V. Foteinos, G. Poulios, V. Stavroulaki, P. Vlacheas, P. Demestichas, A. Baranov, A. Biswas, and R. Giaffreda, "Virtualization and cognitive management of real world objects in the internet of things" in IEEE International Conference on Green Computing and Communications (GreenCom), 2012, pp. 187-194. DOI: 10.1109/GreenCom.2012.37. 